\title{
GW170817: The key to the door of multi-messenger astronomy including gravitational waves
}

\author{
$\mathrm{He} \mathrm{Gao*}$ \\ Department of Astronomy, Beijing Normal University, Beijing 100875, China \\ Received November 30, 2017; accepted December 13, 2017; published online February 26, 2018 \\ Citation: H. Gao, GW170817: The key to the door of multi-messenger astronomy including gravitational waves, Sci. China-Phys. Mech. Astron. 61, \\ 059531 (2018), https://doi.org/10.1007/s11433-017-9149-3
}

On September 14, 2015, the Laser Interferometer Gravitational-wave Observatory (LIGO) team achieved the first-ever direct detection of a gravitational wave $(\mathrm{GW})$ event from a binary black hole (BH) merger (GW150914), indicating the opening of GW observational window [1]. The success of LIGO is due to the tremendous developments in experimental technologies [2-6]. As of October 2017, the LIGO (later joint by VIRGO) team had published 3 additional $\mathrm{BH}$ BH merger events (GW151226, GW170104, GW170814) and one double neutron star (NS) merger (GW170817) gravitational wave event [7-10]. Moreover, in the case of GW170817, many electromagnetic (EM) facilities detected its EM counterparts in multi-wavelengths [11]. This has opened up a new era of GW astronomy, whereby comprehensive analyses of multi-messenger signals will shed light on both GW and EM studies.

GW170817 was first detected online by a single-detector of LIGO-Hanford and was rapidly confirmed by re-analysis of the data from the LIGO-Hanford, LIGO-Livingston, and Virgo detectors with a high coincidence level [10]. Based only on a comprehensive analysis of the GW data, the following properties of the GW source were obtained: the source is located in a region of $28 \mathrm{deg}^{2}$ at a distance of $(40 \pm 8)$ $\mathrm{Mpc}$ and is a compact binary system with a chirp mass of $(1.188 \pm 0.004) M_{\odot}$. Under a high dimensionless NS spin $(\chi<0.89)$ prior, the mass ratio of the two compact objects is in the range 0.4-1.0, such that the estimated masses

\footnotetext{
*Corresponding author (email: gaohe@bnu.edu.cn)
}

of the two objects are $1.36 M_{\odot}<M_{1}<2.26 M_{\odot}$ and $0.86 M_{\odot}<M_{2}<1.36 M_{\odot}$. Under a low dimensionless NS spin $(\chi<0.05)$ prior, the mass ratio of the two compact objects is in the range of $0.7-1.0$, and the estimated masses of these two objects are $1.36 M_{\odot}<M_{1}<1.60 M_{\odot}$ and $1.17 M_{\odot}<M_{2}<1.36 M_{\odot}$, which is consistent with a binary whose components are neutron stars [10].

A weak short-duration gamma-ray burst (SGRB) 170817A from the direction of GW signal was detected $1.7 \mathrm{~s}$ after coalescence by the Fermi-GBM [12] and later confirmed by INTEGRAL [13]. Ten hours later, a group of ground-based optical telescopes detected a fast brightening optical signal in the NGC 4993 galaxy ([11], for a summary). As the distance of NGC 4993 ( $40 \mathrm{Mpc}$ ) agrees with the distance of the GW source, and the optical signal light and spectral evolution behavior were in good agreement with the theoretical predictions, the optical signal was confirmed as a counterpart of GW170817. The Chandra telescope detected a dark X-ray signal with a deep exposure $9 \mathrm{~d}$ later. Considering the observed upper limit given by Swift-XRT, the X-ray signal most likely corresponded to a late brightening signal. Finally, the VLA telescope detected a radio band counterpart $16.2 \mathrm{~d}$ later. The radio signal is still in the brightening phase, consistent with theoretical expectations ([11], for a summary).

These observations were not surprising. For double NS mergers, many associated EM counterparts have already been proposed in the literature and their relative brightness is essentially determined by the properties of the merger remnant. During a merger, a small part of the material is ejected 
nearly isotropically with a sub-relativistic velocity, including the tidally ripped and dynamically launched matter (dynamical ejecta), and the matter launched from the neutrino-driven wind from the accretion disk (disk wind ejecta) ([14], for a review). The remaining material settles to form a new central compact object, either a black hole or a neutron star, depending on its total mass and the NS equation of state [15].

It is generally believed that, for both cases, SGRBs and their afterglow emission are expected as one of the major EM counterparts of NS-NS mergers [16,17]. Moreover, an optical/IR transient is expected to be associated with an NS-NS merger event, as the merger-ejected materials are neutronrich and the radioactivity of these materials heat the ejecta to power the transient [18-20]. When the merger product is a $\mathrm{BH}$, the r-process related radioactivity serves as the only heating source. In this case, the luminosity of the optical/IR transient is $\sim 10^{3}$ times of the nova luminosity [19], and is thus called "kilonova" emission. It is worth noting that the neutron fractions of the dynamical ejecta and the disk wind ejecta are different. For the former, the neutron fraction is high enough to produce heavy nuclei with $A \gtrsim 130$, including the lanthanide and actinide group, so that its opacity is relatively high $[21,22]$. In this case, the dynamical ejecta produce red "kilonova" emission in the near IR band, while the disk wind ejecta produce blue "kilonova" emission in the optical band [23]. When the merger product is a NS, the magnetic spin-down of the NS provides an additional source of sustained energy injection, which easily exceeds the r-process power and significantly enhances both the "red" and "blue" kilonova luminosities by more than one order of magnitude $[24,25]$. Both dynamical and disk wind ejecta interact with the ambient medium. When the merger product is a $\mathrm{BH}$, the interaction generates a weak external shock and provides a long-lasting but weak radio emission component [26]. When the merger product is a NS, the energy injection from the NS accelerates the ejecta to a mildly or even moderately relativistic speed, generating a strong external shock and giving rise to a broad-band afterglow emission [27]. Nevertheless, in the directions with ejecta cavity or after the ejecta becomes transparent, X-rays from direct dissipation of the magnetar wind can escape and reach the observer [28-30].

The joint detection of GW170817 and its multi-band EM counterpart confirms the previous theoretical predictions. The observations of GW170817 and GRB 170817A confirm that the NS-NS merger events are indeed one of the origins of the GRBs. The discovery of the optical/IR counterpart confirms that the NS-NS merger events are indeed an important site for r-process nuclear reactions, which may explain the origin of the heavy elements in the universe. It is interesting to note that the evolution of the observed optical/IR counterpart of GW170817 could be interpreted by invoking a "blue" and a "red" kilonova ([14], and reference therein).

On the other hand, there are still some unexpected sur- prises in the observation of the GW170817 and its EM counterparts, with many unanswered questions suitable for further investigations. For example, in the previous study, it was generally believed that short GRBs were produced by relativistic jets with relatively small opening angles, only detectable in preferred directions. In this case, the probability that a very close GW event, like GW170817, being simultaneously detected with short GRBs should be very low. However, we have detected GRB 170817A associated with GW170817. Nevertheless, the luminosity of GRB 170817A is very small, $\sim 3$ orders of magnitude lower than the normal short GRBs [31]. The X-ray afterglow of GRB 170817A is also very weak in the beginning, but at later times, there is a re-brightening signature in the X-ray band. Considering these properties, it has been proposed that SGRBs may be generated by a structured jet with a larger opening angle [31-35]. In particular, when a relativistic jet propagates through surrounding ejecta, a mildly relativistic cocoon is formed that embraces the main jet. The large opening angle and relatively small velocity of the cocoon greatly enhance the detection probability of the SGRB. For GRB 170817A, our line of sight may fall onto the cocoon direction, so that a relatively weak gamma-ray emission is detected. The late X-ray re-brightening may be due to the off-axis afterglow from the main jet.

Based on the detection of GRB 170817A, one can roughly estimate the event rate density of low luminosity short GRBs, and it can be found that at most a few GRB 170817A-like events should have already been detected by the GBM detector [31]. By investigation, the GBM has indeed detected a number of short GRBs similar to GRB 170817A in terms of flux and signal-to-noise ratio. Do these GRBs come from NSNS mergers? If GRB 170817A is a special short GRBs, can we confirm that those high-luminosity SGRBs also originated from NS-NS mergers? Note that it has long been proposed that NS-BH mergers could also generate SGRBs, increasing the complexity of the situation. More observations in the future will contribute to the further study of these issues.

It is worth noting that for the observations of the EM counterparts of GW170817, Chinese facilities, such as the Hard X-ray Modulation Telescope (HXMT) and the second telescope of the Antarctic Survey Telescopes at Dome A Antarctica (AST3-2), made commendable contributions in $\mathrm{X}$-ray and the optical band [36,37]. In the near future, the Chinese-French cooperation Space Variable Objects Monitor (SVOM) [38] and the Einstein Probe (EP) [39] satellites will be launched, which will play important roles in the future exploration of the gravitational wave electromagnetic counterparts.

In summary, the door to multi-messenger astronomy, including the gravitational wave, has been opened by the GW170817 key. With further improvements of the LIGO sensitivity, increasingly more GW+EM joint detection 
events are expected. Comprehensive analyses of the multimessenger information will help to break the model parameter degeneracy and reduce parameter space, so as to better reveal the physical properties of the merger system and process [40-43]. For instance, it has recently been proposed that the follow up optical/IR observations of GW170817 could provide a more stringent constraint on the mass ratio of the two NSs than that from the analysis using pure GW signals [44]. For NS-NS merger systems, the combination of the GW and EM signals could be used to constrain the EoS of the NS $[15,45]$. The combination of the multi-messenger information could also allow the use of the GW source as a cosmic probe to constrain the cosmological parameters [46], calculate the GW background from the NS-NS mergers [47-49], and test Einstein's equivalence principle, general relativity, and other important physics principles [50-53]. Therefore, we expect that in such a new era of GW astronomy, human exploration of the unknown will inevitably increase.

This work was supported by the National Basic Research Program of China (Grant No. 2014CB845800), the National Natural Science Foundation of China (Grant Nos. 11722324, 11603003, 11633001, and 11690024), and the Strategic Priority Research Program of the Chinese Academy of Sciences (Grant No. XDB23040100).

1 B. P. Abbott, et al. (LIGO Scientific Collaboration and Virgo Collaboration), Phys. Rev. Lett. 116, 061102 (2016).

2 D. Blair, L. Ju, and Z. H. Zhu, Sci. China-Phys. Mech. Astron. 58, 120401 (2015).

3 D. Blair, L. Ju, C. N. Zhao, L. Q. Wen, Q. Chu, Q. Fang, R. G. Cai, J. R. Gao, X. C. Lin, D. Liu, L. A. Wu, Z. H. Zhu, D. H. Reitze, K. Arai, F. Zhang, R. Flaminio, X. J. Zhu, G. Hobbs, R. N. Manchester, R. M. Shannon, C. Baccigalupi, W. Gao, P. Xu, X. Bian, Z. J. Cao, Z. J. Chang, P. Dong, X. F. Gong, S. L. Huang, P. Ju, Z. R. Luo, L. E. Qiang, W. L. Tang, X. Y. Wan, Y. Wang, S. N. Xu, Y. L. Zang, H. P. Zhang, Y. K. Lau, and W. T. Ni, Sci. China-Phys. Mech. Astron. 58, 120402 (2015).

4 D. Blair, L. Ju, C. N. Zhao, L. Q. Wen, H. X. Miao, R. G. Cai, J. R. Gao, X. C. Lin, D. Liu, L. A. Wu, Z. H. Zhu, G. Hammond, H. J. Paik, V. Fafone, A. Rocchi, C. Blair, Y. Q. Ma, J. Y. Qin, and M. Page, Sci. China-Phys. Mech. Astron. 58, 120405 (2015).

5 Z. J. Cao, Sci. China-Phys. Mech. Astron. 59, 110431 (2016).

6 V. P. Mitrofanov, S. Chao, H. W. Pan, L. C. Kuo, G. Cole, J. Degallaix, and B. Willke, Sci. China-Phys. Mech. Astron. 58, 120404 (2015).

7 B. P. Abbott, et al. (LIGO Scientific Collaboration and Virgo Collaboration), Phys. Rev. Lett. 116, 131103 (2016).

8 B. P. Abbott, et al. (LIGO Scientific Collaboration and Virgo Collaboration), Phys. Rev. Lett. 118, 121101 (2017).

9 B. P. Abbott, et al. (LIGO Scientific Collaboration and Virgo Collaboration), Phys. Rev. Lett. 119, 141101 (2017).

10 B. P. Abbott, et al. (LIGO Scientific Collaboration and Virgo Collaboration), Phys. Rev. Lett. 119, 161101 (2017).

11 B. P. Abbott, et al. (LIGO Scientific Collaboration and Virgo Collaboration), Astrophys. J. 848, L13 (2017).

12 A. Goldstein, P. Veres, E. Burns, M. S. Briggs, R. Hamburg, D. Kocevski, C. A. Wilson-Hodge, R. D. Preece, S. Poolakkil, O. J. Roberts, C. M. Hui, V. Connaughton, J. Racusin, A. Kienlin, T. D. Canton, N. Christensen, T. Littenberg, K. Siellez, L. Blackburn, J. Broida, E. Bissaldi, W. H. Cleveland, M. H. Gibby, M. M. Giles, R. M. Kippen, S. McBreen, J. McEnery, C. A. Meegan, W. S. Paciesas, and M. Stanbro, Astrophys. J. 848, L14 (2017).
13 V. Savchenko, C. Ferrigno, E. Kuulkers, A. Bazzano, E. Bozzo, S. Brandt, J. Chenevez, T. J. L. Courvoisier, R. Diehl, A. Domingo, L. Hanlon, E. Jourdain, A. von Kienlin, P. Laurent, F. Lebrun, A. Lutovinov, A. Martin-Carrillo, S. Mereghetti, L. Natalucci, J. Rodi, J. P. Roques, R. Sunyaev, and P. Ubertini, Astrophys. J. 848, L15 (2017).

14 B. D. Metzger, Liv. Rev. Relativ. 20, 3 (2017).

15 H. Gao, B. Zhang, and H. J. Lü, Phys. Rev. D 93, 044065 (2016).

16 D. Eichler, M. Livio, T. Piran, and D. N. Schramm, Nature 340, 126 (1989).

17 R. Narayan, B. Paczynski, and T. Piran, Astrophys. J. 395, L83 (1992).

18 L. X. Li, and B. Paczyński, Astrophys. J. 507, L59 (1998).

19 B. D. Metzger, G. Martínez-Pinedo, S. Darbha, E. Quataert, A. Arcones, D. Kasen, R. Thomas, P. Nugent, I. V. Panov, and N. T. Zinner, Mon. Not. R. Astron. Soc. 406, 2650 (2010).

20 J. Barnes, and D. Kasen, Astrophys. J. 775, 18 (2013).

21 D. Kasen, N. R. Badnell, and J. Barnes, Astrophys. J. 774, 25 (2013).

22 M. Tanaka, and K. Hotokezaka, Astrophys. J. 775, 113 (2013).

23 B. D. Metzger, and R. Fernández, Mon. Not. R. Astron. Soc. 441, 3444 (2014).

24 Y. W. Yu, B. Zhang, and H. Gao, Astrophys. J. 776, L40 (2013).

25 B. D. Metzger, and A. L. Piro, Mon. Not. R. Astron. Soc. 439, 3916 (2014).

26 E. Nakar, and T. Piran, Nature 478, 82 (2011).

27 H. Gao, X. Ding, X. F. Wu, B. Zhang, and Z. G. Dai, Astrophys. J. 771, 86 (2013).

28 B. Zhang, Astrophys. J. 763, L22 (2013).

29 H. Gao, X. Ding, X. F. Wu, Z. G. Dai, and B. Zhang, Astrophys. J. 807, 163 (2015).

30 H. Sun, B. Zhang, and H. Gao, Astrophys. J. 835, 7 (2017).

31 B.-B. Zhang, B. Zhang, H. Sun, W.-H. Lei, H. Gao, Y. Li, L. Shao, Y. Zhao, Y.-D. Hu, H.-J. L , X.-F. Wu, X.-L. Fan, G. Wang, A. J. Castro-Tirado, S. Zhang, B.-Y. Yu, Y.-Y. Cao, and E.-W. Liang, arXiv: 1710.05851.

32 O. Gottlieb, E. Nakar, T. Piran, and K. Hotokezaka, arXiv: 1710.05896.

33 M. M. Kasliwal, E. Nakar, L. P. Singer, D. L. Kaplan, D. O. Cook, A. Van Sistine, R. M. Lau, C. Fremling, O. Gottlieb, J. E. Jencson, S. M. Adams, U. Feindt, K. Hotokezaka, S. Ghosh, D. A. Perley, P. C. Yu, T. Piran, J. R. Allison, G. C. Anupama, A. Balasubramanian, K. W. Bannister, J. Bally, J. Barnes, S. Barway, E. Bellm, V. Bhalerao, D. Bhattacharya, N. Blagorodnova, J. S. Bloom, P. R. Brady, C. Cannella, D. Chatterjee, S. B. Cenko, B. E. Cobb, C. Copperwheat, A. Corsi, K. De, D. Dobie, S. W. K. Emery, P. A. Evans, O. D. Fox, D. A. Frail, C. Frohmaier, A. Goobar, G. Hallinan, F. Harrison, G. Helou, T. Hinderer, A. Y. Q. Ho, A. Horesh, W. H. Ip, R. Itoh, D. Kasen, H. Kim, N. P. M. Kuin, T. Kupfer, C. Lynch, K. Madsen, P. A. Mazzali, A. A. Miller, K. Mooley, T. Murphy, C. C. Ngeow, D. Nichols, S. Nissanke, P. Nugent, E. O. Ofek, H. Qi, R. M. Quimby, S. Rosswog, F. Rusu, E. M. Sadler, P. Schmidt, J. Sollerman, I. Steele, A. R. Williamson, Y. Xu, L. Yan, Y. Yatsu, C. Zhang, and W. Zhao, Science 358, 1559 (2017).

34 A. L. Piro, and J. A. Kollmeier, arXiv: 1710.05822.

35 D. Xiao, L.-D. Liu, Z.-G. Dai, and X.-F. Wu, arXiv: 1710.05910.

36 T. P. Li, S. L. Xiong, S. N. Zhang, F. J. Lu, L. M. Song, X. L. Cao, Z. Chang, G. Chen, L. Chen, T. X. Chen, Y. Chen, Y. B. Chen, Y. P. Chen, W. Cui, W. W. Cui, J. K. Deng, Y. W. Dong, Y. Y. Du, M. X. Fu, G. H. Gao, H. Gao, M. Gao, M. Y. Ge, Y. D. Gu, J. Guan, C. C. Guo, D. W. Han, W. Hu, Y. Huang, J. Huo, S. M. Jia, L. H. Jiang, W. C. Jiang, J. Jin, Y. J. Jin, B. Li, C. K. Li, G. Li, M. S. Li, W. Li, X. Li, X. B. Li, X. F. Li, Y. G. Li, Z. J. Li, Z. W. Li, X. H. Liang, J. Y. Liao, C. Z. Liu, G. Q. Liu, H. W. Liu, S. Z. Liu, X. J. Liu, Y. Liu, Y. N. Liu, B. Lu, X. F. Lu, T. Luo, X. Ma, B. Meng, Y. Nang, J. Y. Nie, G. Ou, J. L. Qu, N. Sai, L. Sun, Y. Tan, L. Tao, W. H. Tao, Y. L. Tuo, G. F. Wang, H. Y. Wang, J. Wang, W. S. Wang, Y. S. Wang, X. Y. Wen, B. B. Wu, M. Wu, G. C. Xiao, H. Xu, Y. P. Xu, L. L. Yan, J. W. Yang, S. Yang, Y. J. Yang, A. M. Zhang, C. L. Zhang, C. M. Zhang, F. Zhang, H. M. Zhang, J. Zhang, Q. Zhang, S. Zhang, T. Zhang, W. Zhang, W. C. Zhang, W. Z. Zhang, Y. Zhang, Y. Zhang, Y. F. Zhang, Y. J. Zhang, Z. Zhang, Z. L. Zhang, H. S. Zhao, J. L. Zhao, X. F. Zhao, S. J. Zheng, Y. Zhu, Y. 
X. Zhu, and C. L. Zou, Sci. China-Phys. Mech. Astron. 61, 031011 (2018).

37 L. Hu, X. Wu, I. Andreoni, M. C. B. Ashley, J. Cooke, X. Cui, F. Du, Z. Dai, B. Gu, Y. Hu, H. Lu, X. Li, Z. Li, E. Liang, L. Liu, B. Ma, Z. Shang, T. Sun, N. B. Suntzeff, C. Tao, S. A. Uddin, L. Wang, X. Wang, H. Wen, D. Xiao, J. Xu, J. Yang, S. Yang, X. Yuan, H. Zhou, H. Zhang, J. Zhou, and Z. Zhu, Sci. Bull. 62, 1433 (2017).

38 J. Wei, B. Cordier, S. Antier, P. Antilogus, J.-L. Atteia, A. Bajat, S. Basa, V. Beckmann, M.G. Bernardini, S. Boissier, L. Bouchet, V. Burwitz, A. Claret, Z.-G. Dai, F. Daigne, J. Deng, D. Dornic, H. Feng, T. Foglizzo, H. Gao, N. Gehrels, O. Godet, A. Goldwurm, F. Gonzalez, L. Gosset, D. G?tz, C. Gouiffes, F. Grise, A. Gros, J. Guilet, X. Han, M. Huang, Y.-F. Huang, M. Jouret, A. Klotz, O. La Marle, C. Lachaud, E. Le Floch, W. Lee, N. Leroy, L.-X. Li, S. C. Li, Z. Li, E.-W. Liang, H. Lyu, K. Mercier, G. Migliori, R. Mochkovitch, P. O’Brien, J. Osborne, J. Paul, E. Perinati, P. Petitjean, F. Piron, Y. Qiu, A. Rau, J. Rodriguez, S. Schanne, N. Tanvir, E. Vangioni, S. Vergani, F.-Y. Wang, J. Wang, X.-G. Wang, X.-Y. Wang, A. Watson, N. Webb, J. J. Wei, R. Willingale, C. Wu, X.-F. Wu, L.-P. Xin, D. Xu, S. Yu, W.-F. Yu, Y.-W. Yu, B. Zhang, S.-N. Zhang, Y. Zhang, and X. L. Zhou, arXiv: 1610.06892.

39 W. M. Yuan, et al. (the Einstein Probe team), arXiv: 1506.07735.

40 X. L. Fan, and M. Hendry, arXiv: 1509.06022.

41 X. L. Fan, Sci. China-Phys. Mech. Astron. 59, 640001 (2016).
42 X. L. Fan, C. Messenger, and I. S. Heng, Phys. Rev. Lett. 119, 181102 (2017).

43 H. M. Lee, E. O. Le Bigot, Z. H. Du, Z. X. Lin, X. Y. Guo, L. Q. Wen, K. S. Phukon, V. Pandey, S. Bose, X. L. Fan, and M. Hendry, Sci. China-Phys. Mech. Astron. 58, 120403 (2015).

44 H. Gao, Z. Cao, S. Ai, and B. Zhang, arXiv: 1711.08577.

45 A. Li, B. Zhang, N. B. Zhang, H. Gao, B. Qi, and T. Liu, Phys. Rev. D 94, 083010 (2016)

46 K. Liao, X.-L. Fan, X.-H. Ding, M. Biesiada, and Z.-H. Zhu, arXiv: 1703.04151.

47 X. J. Zhu, E. Howell, T. Regimbau, D. Blair, and Z. H. Zhu, Astrophys. J. 739, 86 (2011).

48 X. J. Zhu, X. L. Fan, and Z. H. Zhu, Astrophys. J. 729, 59 (2011).

49 X. J. Zhu, E. J. Howell, D. G. Blair, and Z. H. Zhu, Mon. Not. R. Astron. Soc. 431, 882 (2013).

50 B. P. Abbott, et al. (LIGO Scientific Collaboration and Virgo Collaboration), Nature 551, 85 (2017).

51 X. L. Fan, K. Liao, M. Biesiada, A. Piórkowska-Kurpas, and Z. H. Zhu, Phys. Rev. Lett. 118, 091102 (2017).

52 J.-J. Wei, B.-B. Zhang, X.-F. Wu, H. Gao, P. Mészáros, B. Zhang, Z.-G. Dai, S.-N. Zhang, and Z.-H. Zhu, arXiv: 1710.05860.

53 X. F. Wu, H. Gao, J. J. Wei, P. Mészáros, B. Zhang, Z. G. Dai, S. N. Zhang, and Z. H. Zhu, Phys. Rev. D 94, 024061 (2016). 